\title{
Signal processing for ultrasonic clamp-on-sensor systems
}

\author{
Sebastian Wöckel $^{1}$, Ulrike Hempel ${ }^{1}$, Jörg Auge ${ }^{2}$ \\ ${ }^{1}$ Measuring \& Analyser Systems, Institute for Automation and Communication e.V., Werner- \\ Heisenberg-Str. 1, 39106 Magdeburg, Germany \\ ${ }^{2}$ Magdeburg-Stendal University of Applied Sciences, Magdeburg, Germany \\ Email: sebastian.woeckel@ifak.eu
}

\begin{abstract}
:
The paper addresses several techniques of signal processing in case of non-invasive ultrasonic applications. In first the signal oriented modelling of linear systems demonstrates the estimation of wave propagation and mode conversion in layered media. Its reliability in case of curvilinear surfaces, divergent sound fields and non-perpendicular incident with additional interface effects is discussed. In extension the paper deals with the potential of coding techniques to substitute any arbitrary short pulse signal with increased signal to noise ratio in case of high attenuation and reflection loss. The aspects of coded signals will be exemplified on measurement results concerning structure borne sound with dispersion and sound propagation in high attenuating environments. Finally, the statistical processing and the parameter estimation are demonstrated on particular and layered systems.
\end{abstract}

Key words: signal processing, ultrasonic measurement, parameter estimation, linear modeling

\section{Motivation}

Ultrasonic measurements are common techniques in industrial liquid analysis. Especially the non-invasive clamp-on configuration is an interesting approach due to its non-destructive nature and easy handling for level or flow measurement. Such a sensor can be mounted on existing reactors or pipes without either stopping the process or rebuilding the system. However, the applicability of clamp-on systems often depends on a sophisticated signal analysis in order to discriminate the measurement effect from disturbances caused by the multimodal sound propagation, misalignment of the transducer or undefined coupling and intermediate layers with impedance mismatch. In particular the additional intermediate layer (pipe wall) reduces the signal to noise ratio due to reflection loss and mode conversion.

Therefore this paper addresses several techniques of signal processing which are applied to overcome such difficulties. At first the signal oriented modelling of linear systems demonstrates the estimation of wave propagation and mode conversion in layered media. Its reliability in case of curvilinear surfaces, divergent sound fields and non-perpendicular incident with additional interface effects is discussed. Further, this kind of signal oriented modelling, without fully considering the physical behaviour, enables the possibility of autonomous numerical optimization towards estimation of a large num- ber of unknown parameters or statistical features and calibration tasks.

Additionally, the paper deals with the potential of coding techniques to substitute any arbitrary short pulse signal with increased signal to noise ratio in case of high attenuation and reflection loss. Finally, the benefit of statistical processing in case of level detection is discussed.

\section{Ultrasonic clamp-on system parameter}

The main aspect of a robust non-intrusive sensor system is the knowledge on the multimodal wave propagation in pipes filled with fluids. According to the changing acoustic parameters the pipe wall (or any other solid interface) influences the transmission, reflection and mode conversion of any acoustic wave. Usually the conversion of the longitudinal and transversal primary wave modes, which are mainly used for transmission measurements, can simply be described with a reflection $R$ and transmission factor $D(\alpha-$ attenuation, $c$ - sound velocity, $\rho$ density):

$$
\begin{aligned}
& R_{\alpha_{e}}=\frac{\cos \alpha_{e}-\left(\varrho_{1} c_{1} / \varrho_{2} c_{2}\right) \sqrt{1-\left(c_{2} / c_{1}\right)^{2} \sin ^{2} \alpha_{e}}}{\cos \alpha_{e}+\left(\varrho_{1} c_{1} / \varrho_{2} c_{2}\right) \sqrt{1-\left(c_{2} / c_{1}\right)^{2} \sin ^{2} \alpha_{e}}} \\
& D_{\alpha_{e}}=\frac{2 \cos \alpha_{e}}{\cos \alpha_{e}+\left(\varrho_{1} c_{1} / \varrho_{2} c_{2}\right) \sqrt{1-\left(c_{2} / c_{1}\right)^{2} \sin ^{2} \alpha_{e}}}
\end{aligned}
$$

In consequence of the acoustic contrast of the reverberative pipe wall to the fluid with low im- 
pedance there is no critical angle for the genesis of surface acoustic modes (Fig. 1). Further the high impedance solid layer (pipe wall) focuses and widens the sonic field due to the low angle of beam $\left(\beta_{\mathrm{L}}\right)$. Thus a planar wave front inside the pipe can be assumed (Fig. 2) and the resulting directivity of the pipe wall enhances the use of the linear model.

The law of refraction (1) is also valid for the outer pipe interface since the impedance of the delay line and its sort of strict or loose coupling is regarded. A strict coupling would evoke secondary waves travelling along the pipe wall, which are not described with (1). Because of the low incident angle, of a transducer with high directivity, Rayleigh or creeping waves can be neglected. In certain studies [1] it could be shown, that Lamb-waves have a high damping for liquid filled pipes because of the contrast in impedance between the inner and outer pipe interface. Therefore the structural borne sound needs to be regarded for small transmitterreceiver-distances or gas filled pipes, only. In case of typical measuring distances the quasilongitudinal mode [1] or multi reflection path dominate the signal.

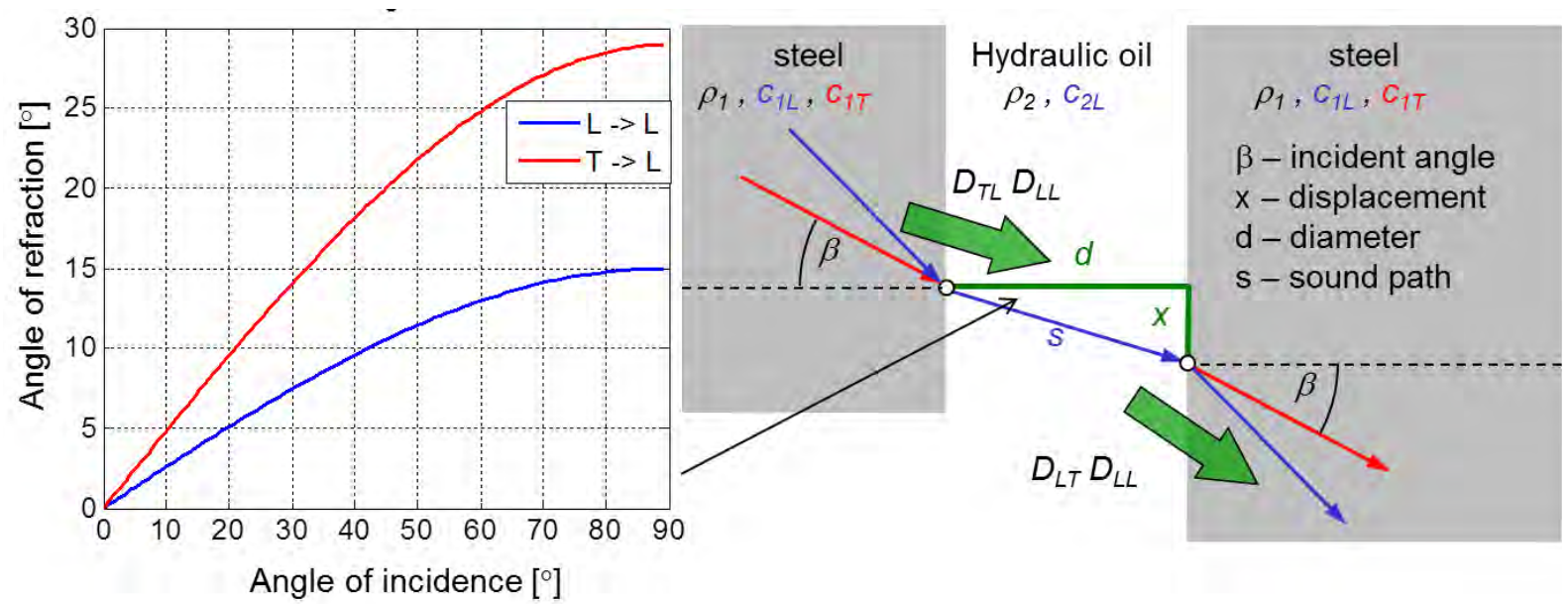

Figure 1: Model of diametric transmission and angle of refraction within low impedance medium (oil) in case of longitudinal ( $L$ / blue) or transversal ( $T$ / red) waves impinging from the high impedance pipe wall (steel).
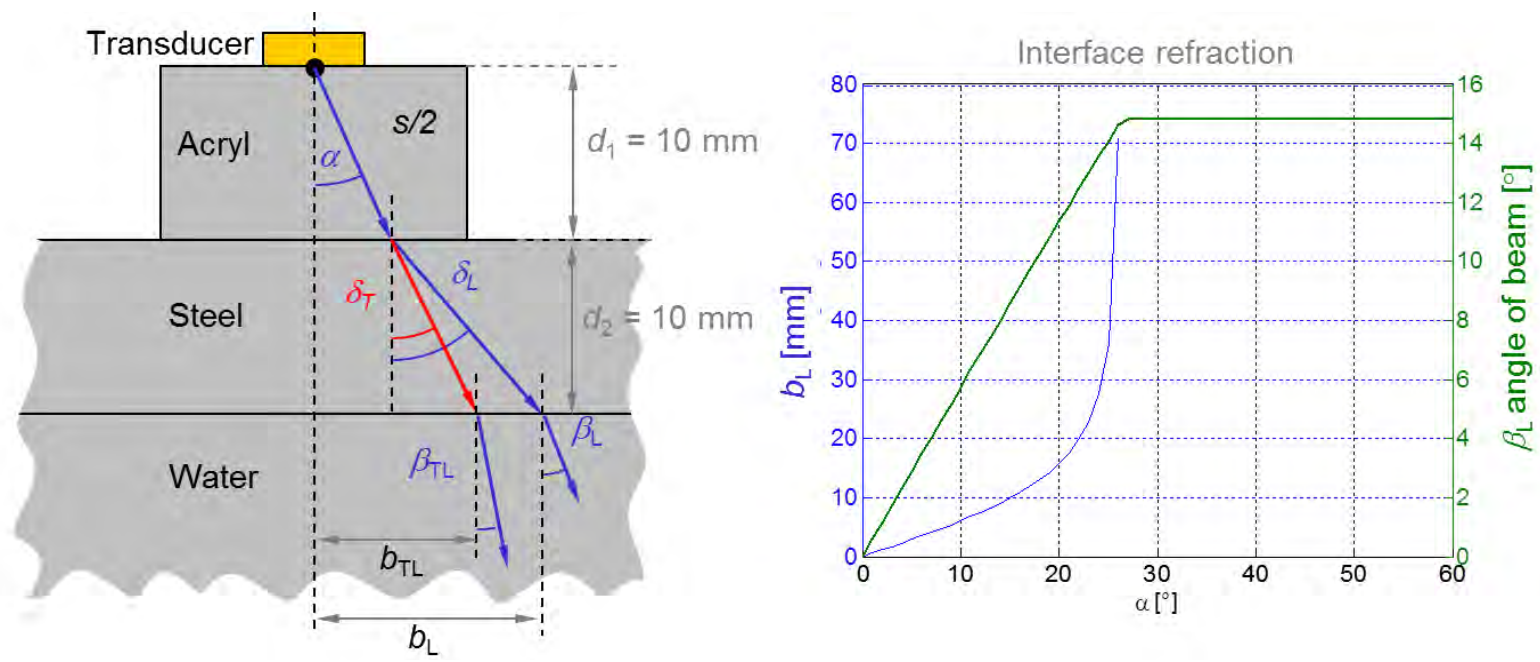

Figure 2: Schematic of the (linear model) sound transmission through a delay line and pipe wall into a liquid exemplifying the basic assumption on the focusing and linearizing effect on the divergent wave field (acryl: $c_{L}=2567 \mathrm{~m} / \mathrm{s}, c_{T}=1450 \mathrm{~m} / \mathrm{s}$; steel: $c_{L}=5790 \mathrm{~m} / \mathrm{s}, c_{T}=3100 \mathrm{~m} / \mathrm{s} ;$ water: $c_{L}=1480 \mathrm{~m} / \mathrm{s}$ ).

According to these assumptions the main acoustic (measurable) longitudinal and transversal transmission modes and their transmission, reflection and conversion can be described in a closed form [1] using the matrix formulation [2] of a linear Mason-model [3] based on the signal flow graph. Herein the graph can be separated in modules represent- ing the propagation within a homogeneous layer and modules representing the interface behavior.

\section{Linear model}

Regarding a pipe cross section filled with a homogeneous medium and assuming a lateral dimension of the material under test larger than 
the diameter of the transducers the transmission can be reduced to a one dimensional model with layer thicknesses $d_{i}$ (Fig. 3). This kind of modelling is valid since a narrow radiation pattern [1] can be assumed. Due to that simplification a signal oriented graph can be used to describe the propagation of ultrasonic waves and the medium and pipe dependent measurement effects. Therein all signal paths between the transmitter and the receiver are represented by a Mason-graph [3].

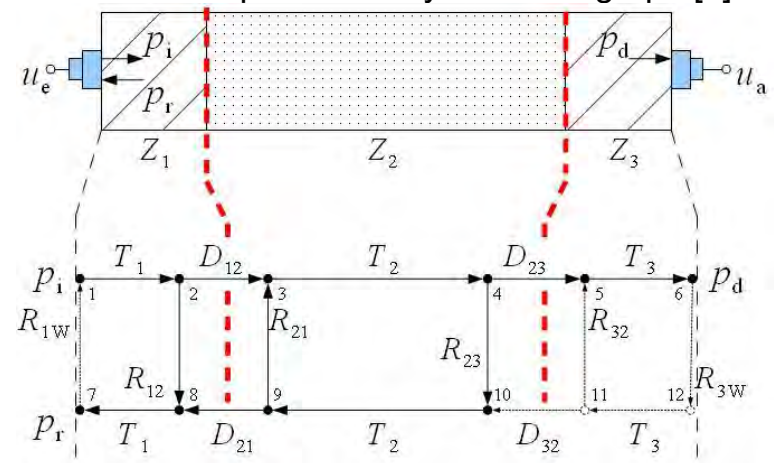

Figure 3: Draft of the measurement setup: pipe cross section with ultrasonic transducers in clamp-on configuration and linear model for simulating the pressure amplitudes (p).

This model includes the emitters and receivers voltage signal, the coefficients of the physical transmission $\left(T_{i}\right)$ within the layers and the reflection $\left(\Gamma_{i}\right)$ at the acoustic interfaces. The transformation of the signal by the transducers is excluded since only two wave modes are used. Based on that Mason-model an analytical solution can be derived to calculate either the transmitted or the reflected signal. Considering all paths in the graph (Fig. 3) for a homogeneous medium the transmission function $G_{T}$ can be derived, which describes the wave propagation for perpendicular incident angle $\alpha$ :

$$
G_{M, T}=\frac{G_{1, T} \Delta_{1, T}}{\Delta}=\frac{T_{1} T_{2} T_{3} D_{12} D_{23}}{1+T_{2}^{2} R_{12} R_{23}}
$$

Concerning varying incident angle, the signal graph has to be generalized where the scalar transmission and reflection functions $T$ and $R$ have to be replaced with tensors $\underline{T}$ and $\underline{R}$ including the conversion and superposition of longitudinal and transversal waves:

$\underline{\underline{T}} \cdot \underline{\boldsymbol{a}}=\left[\begin{array}{ll}T_{L L} & T_{L T r} \\ T_{T r L} & T_{T r T r}\end{array}\right] \cdot\left[\begin{array}{l}a_{L} \\ a_{T r}\end{array}\right]=\left[\begin{array}{l}b_{L} \\ b_{T r}\end{array}\right]$,

Herein $a$ and $b$ represent the generalized impinging and transmitted signal.

Fig. 4 represents the extended signal flow graph using the simple model from Fig. 3 once more, now including the mode conversion and propagation of both wave types. The indexes specify the direction of the transmission and reflection path and the conversion. With the angle dependent acoustic length of the layer thickness

$$
d_{\angle}=\frac{d_{\perp}}{\cos \varphi}
$$

the transmission function becomes:

$$
T=e^{-j \omega \frac{d_{L}}{c}-\alpha d_{L}}
$$

This signal flow graph can be generalized for an infinite number of $N$-layers using the matrix formulation again [2] and separating the graph in modules representing the propagation within a homogeneous layer (Layer-matrix) and modules representing the interface behavior (Conversion-matrix) and junction between adjacent layers (Fig. 5). In case of a solid-liquid interface the Conversion-matrix is sparsely populated. Fig. 6 exemplifies the Layer-matrix of a solid with the functional weights $q_{i}$ and the corresponding system of algebraic equations in matrix form for the longitudinal mode.

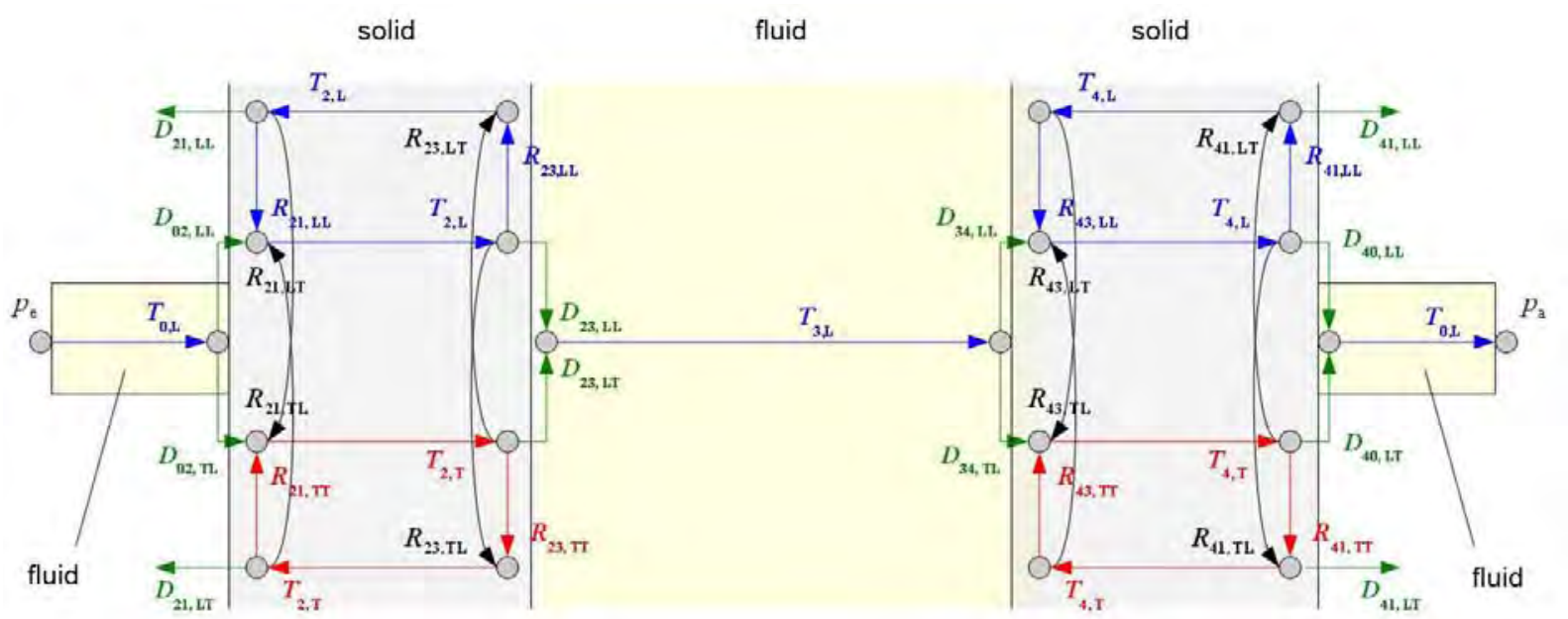

Figure 4: Enhanced signal flow graph of clamp-on transmission with arbitrary incident angle. 


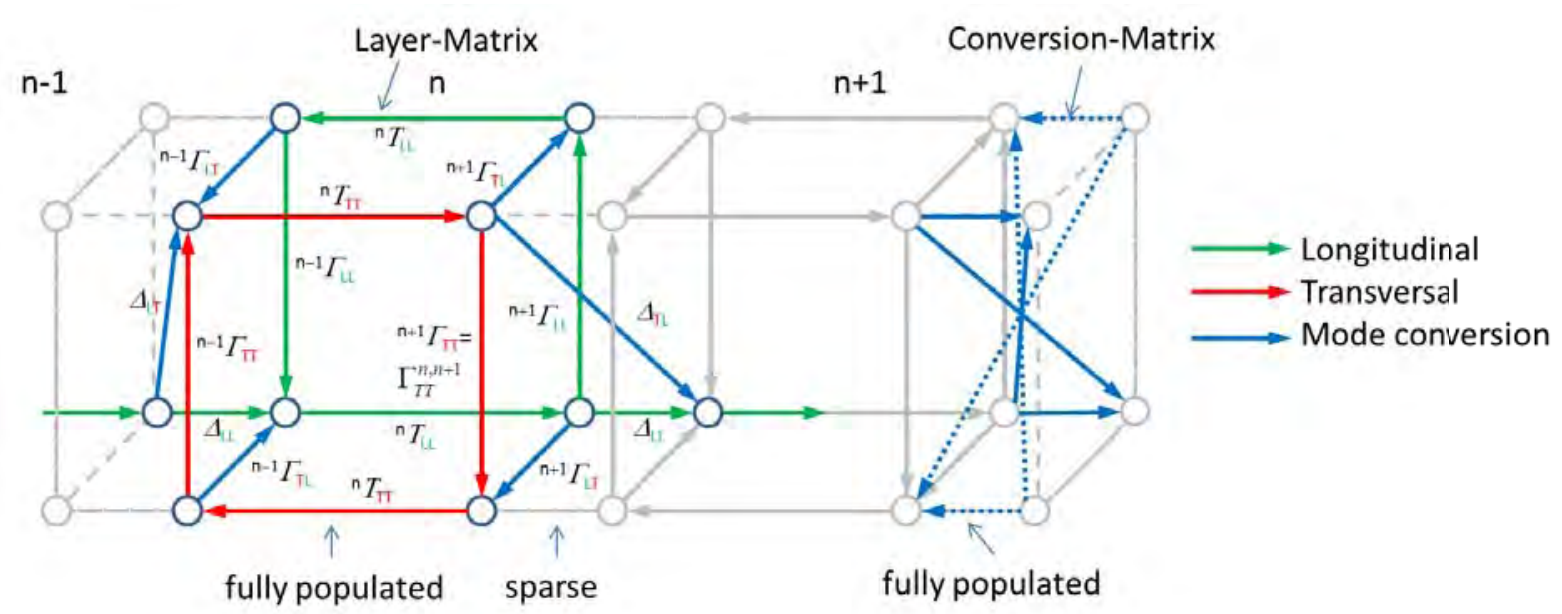

Figure 5: Generalized matrix signal flow graph of an N-layer system.

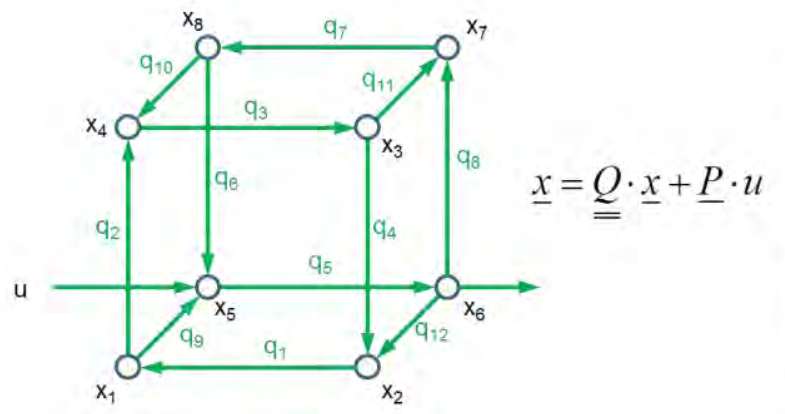

$$
\left[\begin{array}{l}
x_{1} \\
x_{2} \\
x_{3} \\
x_{4} \\
x_{5} \\
x_{6} \\
x_{7} \\
x_{8}
\end{array}\right]=\left[\begin{array}{cccccccc}
0 & q_{1} & 0 & 0 & 0 & 0 & 0 & 0 \\
0 & 0 & q_{4} & 0 & 0 & q_{12} & 0 & 0 \\
0 & 0 & 0 & q_{3} & 0 & 0 & 0 & 0 \\
q_{2} & 0 & 0 & 0 & 0 & 0 & 0 & q_{10} \\
q_{9} & 0 & 0 & 0 & 0 & 0 & 0 & q_{6} \\
0 & 0 & 0 & 0 & q_{5} & 0 & 0 & 0 \\
0 & 0 & q_{11} & 0 & 0 & q_{8} & 0 & 0 \\
0 & 0 & 0 & 0 & 0 & 0 & q_{7} & 0
\end{array}\right] \cdot\left[\begin{array}{c}
x_{1} \\
x_{2} \\
x_{3} \\
x_{4} \\
x_{5} \\
x_{6} \\
x_{7} \\
x_{8}
\end{array}\right]+\left[\begin{array}{l}
0 \\
0 \\
0 \\
0 \\
1 \\
0 \\
0 \\
0
\end{array}\right] \cdot u
$$

Figure 6: Example of Layer-matrix of a solid (left) and unimodal system of algebraic equations (right).

Verification of analytical model: The equation (2) describes the unimodal wave propagation (longitudinal) in boresight direction and neglects all kinds of mode conversion due to nonperpendicular incident angle. This modelling has been verified with the ultrasonic transmission on a metallic steel pipe filled with water. Corresponding to the draft in Fig. 3 two ultrasonic transducers (V323-SU Panametrics) are placed in transmission setup via a delay line on the surface of the steel pipe. In the measurements a pulse excitation with a centre frequency of $f=2.25 \mathrm{MHz}, \quad$ a $10 \mathrm{~dB}$-bandwidth of $B=2 \mathrm{MHz}$ and a sampling frequency of $f_{T}=50 \mathrm{MHz}$ was used for signal detection. Fig.
7 shows the comparison of the raw signals of the model derived with (2) and the measurement. Regardless of angle beam, spreading loss, curvilinear interfaces, thermal drift in wave speed and uneven pipe wall thickness the first time arrival is estimated with $99 \%$ coherence [1], and the multiple ringing in the pipe wall with a precision of $>90 \%$. However, the measurements in addition to calculations proof that in case of perpendicular incident angle, narrow radiation pattern and homogeneous media (even multiphase media) neither crucial disturbing waves caused by transversal mode conversions nor angular signal path appear.

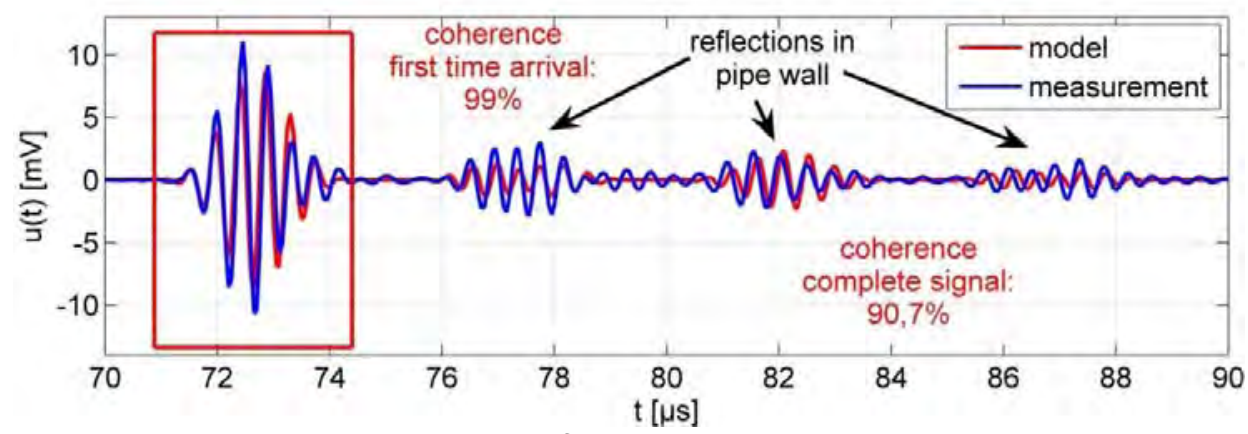

Figure 7: Model solution and measurement of an ultrasonic pulse-transmission on a steel pipe $(d=$ $13 \mathrm{~mm})$ filled with water $(d=100 \mathrm{~mm})$ with V232-SU transducer $(f=2.25 \mathrm{MHz})$. 
The coherence between model and measurement do not decrease significant in case of small arbitrary incident angle and the enhanced signal flow graph with additional transversal modes according to Fig. 4. However this accuracy is only kept with small incident angle, since the model assumes infinite transducers and does not regard the displacement of the wave front due to multiple ringing. Its accuracy degrades rapidly in relation to the transducers area, increasing angle of incident and the reflectivity of single layers. Consequently, the time window with no ambiguity depends on the application.
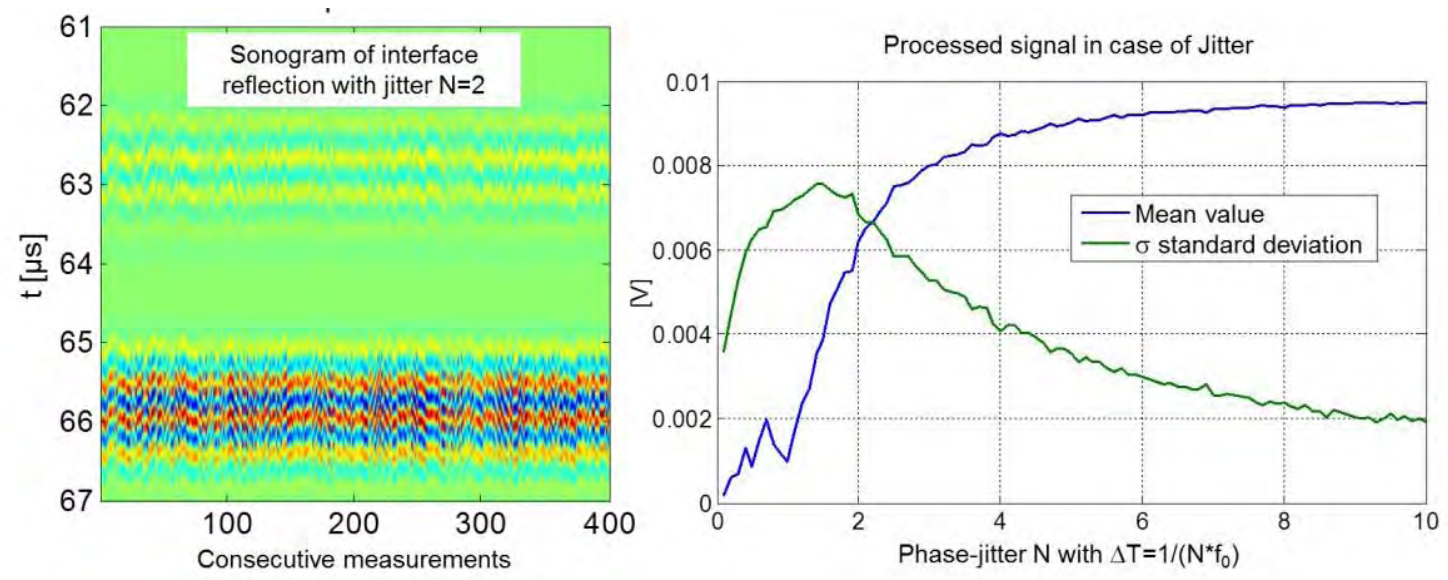

Figure 8: Sonogram of consecutive ultrasonic reflections $\left(f_{0}=2,25 \mathrm{MHz}\right)$ at a static interface with jitter of phase and amplitude (left) and amplitudes of standard deviation and mean value of a number of $N$ stationary reflections in case of a synthetic phase-jitter $\Delta T$ in relation to the period of the signals center frequency (right).

Parameter estimation: Equation (2) represents an analytical form of the wave propagation within the layered media. Since all additional transformations like the transducers transmit $(T x)$ and receive $(R x)$ functions are known, the received transfer signal $\underline{s}(\omega)$ can be calculated in frequency domain with:

$$
\underline{s}(\omega)=R x(\omega) \cdot G_{T}(\omega) \cdot T x(\omega) \cdot \underline{u}(\omega)
$$

This received signal $\underline{s}(\omega)$ represents the impulse response function (IRF) of the whole system which contains the information on the system. Hence this analytical description can be used to characterize the system via an optimization algorithm, which estimates all acoustic relevant parameters.

Investigation on different deterministic [4], stochastic and evolutional [5] optimization algorithm lead to the conclusion, that a hybrid combination of a deterministic (Nelder-MeadSimplex) and an evolutional algorithm suites most needs for system calibration in case of simple clamp-on applications like level detection or non-invasive flow measurements. The algorithm was mainly used to estimate unknown or varying physical parameter of the pipe wall as well as missing or incomplete data on densities and sound velocities.

In general the model-based estimation of those missing parameters can be done by applying a calibration measurement and using the algorithm to fit the model solution to the calibration data by varying the physical input parameters.
For choosing the algorithm in case of ultrasonic clamp-on-systems and the corresponding linear model equation the algorithm needs to be applicable for non-differentiable multidimensional function. Since the solution of the inverse problem is non-linear and ambique the algorithm should converge to the global minimum with a reduced number of algorithm related control parameters.

\section{Signal coding and concept of equivalence}

The electrical excitation signal determines the effectiveness of the transducer, regarding spatial resolution in combination with reduction of thermal loss and increased electro-mechanic coupling factor. In this context typical impulse signals cannot be adapted completely to the transducers frequency spectra, especially due to the maximum power limit. Alternatively a quasiperiodic sequence with orthogonal code can be used [6] to fit the transducers spectrum and to spread the signals energy with a low crest-factor over the whole measurement time window. In case of a linear system the coded signal $m(t)$ can be designed towards its correlation $\Psi_{\mathrm{xy}}$ to be equivalent to the spectra of a system response $y(t)$ of a conventional excitation impulse

$\Psi_{x y}(t)=g(t) * m(t) * m(-t) \approx g(t) * x(t)$

Building on that concept of equivalence, that any arbitrary short time impulse can mathemat- 
ically be replaced by a coded periodic signal, it is possible to improve the transducers excitation behavior and reduce the thermal loss.

\section{Statistical evaluation for ultrasonic level detection}

Common values of processing techniques which are often used for amplitude and travel time measurements are the mean, median or the envelope of raw or correlated signals. In many cases these values are quite robust, but there are also applications where the applicability is not given due to a low signal to noise ratio or correlated noise effects. Since there is a measuring time which allows the processing of a number of signals, alternatively a statistical processing may be applied. Examples are the analysis of frequency components, the sound velocity measurement by using the reflection on particles [7] or inhomogeneous tissue [8] or the particle analysis itself based on a statistical approach [9]. Such techniques can be adapted to quite simpler task of amplitude or travel time measurements for level detection or media analysis.

The basic idea, which is used here, is, that the standard deviation of a randomly changing acoustic system correlates to the amplitude of the single signal itself and may be used instead. For proof of example Fig. 8 shows the acoustic reflections at the static interfaces between water, acryl and air. The signal is synthetically noised with a jitter in amplitude and phase. Obviously with rising jitter (decreasing $N$ ) the mean value decreases. In the range of a phasejitter $N<2$ over the half of the period the maximum of the standard deviation has a higher value than the mean value and thus be more suitable for threshold detection and travel time estimation by maximum value of envelope.

The standard deviation caused by the variation of the amplitude (varying reflectivity) does not improve the interface detection in comparison to the mean value. The phase jitter in relation to the period of the signal is a more suitable parameter. Using a synthetic jitter one can emphasizes the usage of the standard deviation.

\section{Conclusion}

The aspects of linear modelling and parameter estimation where discussed in order to enhance the reliability of ultrasonic clamp-on systems under varying process conditions and unknown physical parameters. In many applications, if no explicit radiation pattern or structure born sound need to be assumed, the linear modelling suffices to provide an enhanced signal analysis [1]. In particular the matrix formulation of the signal flow graph and the automatic computation of a transfer function are helpful to estimate the propagation and conversion due to refraction of combined longitudinal and transversal modes in an infinite layer system.

Adjacent the signal coding and statistical processing addresses the fact of reflection loss and applicability in case of low signal to noise ratio. In detail the equivalent periodic coding has an underachieved potential concerning optimization of ultrasonic systems. The statistical feature extraction as well is useful in case of randomly changing process condition or even may be induced with synthetic noise of the excitation.

In summary, the applicability of the mentioned approaches always depends on the application and may not be generalized. But in conclusion a combination of all processing steps should be sufficient to characterize an ultrasonic noninvasive system and is suitable to support the measurement and accelerate and ensure the engineering process.

\section{References}

[1] S. Wöckel, U. Hempel, J. Auge: Wave propagation phenomena in case of clamp-on ultrasonic systems applied on liquid filled pipes, Sensor+Test Conferences 2011. S. 153-158, CD: ISBN 978-3-9810993-9-3

[2] Chen, H.: The Matrix Expression of Signal Flow Graph and its application in system analysis software, Chinese Journal of Electronics 11 (2002), Nr. 3, S. 361-364

[3] S.J Mason: Further Properties of Signal Flow Graphs, Proc. Institute of Radio Engineers, vol. 44, number 7., 1956, S. 920-926

[4] Z. Michalewicz: How to solve it: Modern Heuristics. Springer, 2004

[5] J. Kacprzyk: Differential evolution: A handbook for global permutation-based combinatorial optimization. Springer, 2009

[6] H. Alrutz: Über die Anwendung von Pseudorauschfolgen zur Messung an linearen Übertragungs-systemen. Dissertation, GeorgAugust-Universität Göttingen, 1983.

[7] M. Lenz, E. Kühnicke, et.al: Sound velocity measurements in fluids using echo signals from scattering particles, ISUD8, September 19-21, 2012, Dresden, Germany

[8] G. Ghoshal, M.L. Oelze, Time domain attenuation estimation method from ultrasonic backscattered signals, J Acoust. Soc. Am. 132 (2012) 533-543

[9] R. Weser, S. Wöckel, B. Wessely, U. Hempel, Particle characterization in highly concentrated dispersions using ultrasonic backscattering method, Ultrasonics (2012), http://dx.doi.org/10.1016/j.ultras.2012.10.013 